\title{
QUALIDADE DE VIDA DE PESSOAS COM DOENÇA CRÔNICA
}

\author{
Luciana Mendes Martins* \\ Ana Paula Dias França* \\ Miako Kimura**
}

MARTINS, L.M.; FRANÇA, A.P.D. ; KIMURA, M. Qualidade de vida de pessoas com doença crônica. Rev Latino-am.enfermagem, Ribeirão Preto, V. 4, n. 3, p. 5-18, dezembro de 1996.

O presente estudo teve como objetivos; identificar o significado de qualidade de vida para pessoas com doença crônica e verificar a interferência da doença sobre a qualidade de vida das mesmas. Foram entrevistadas 71 pessoas de ambos os sexos, com idade entre 13 e 79 anos, atendidas nos ambulatórios de Clínica Médica de 2 hospitais governamentais. Utilizou-se, para coleta de dados, um formulário com questões abertas e fechadas. Os diagnósticos mais freqüentes foram hipertensão arterial e diabetes mellitus. O significado de qualidade vida para as pessoas entrevistadas relacionou-se principalmente ao bem-estar material (40,9\%), ao bemestar físico (23,9\%) e ao bem-estar emocional (11,2\%). A doença crônica interferiu na qualidade de vida das pessoas por alterar, sobretudo, a sua capacidade física (67,6\%), o trabalho/estudo/atividades/ do lar (64,8\%) e a auto-estima (53,5\%). A partir dos resultados obtidos, enfatiza-se a importância de se considerar a multidimensionalidade do conceito de qualidade de vida na assistência de enfermagem as pessoas com doenças crônicas.

UNITERMOS: doença crônica, qualidade de vida

\footnotetext{
* Alunas do $7^{\circ}$ semestre do Curso de Graduação da Escola de Enfermagem da Universidade de São Paulo (EEUSP)

** Enfermeira. Professor Doutor do Departamento de Enfermagem Médico-Cirúrgica da Escola de Enfermagem da Universidade de São Paulo (EEUSP)
} 


\section{I - INTRODUÇÃO}

A crescente evolução tecnológica na assistência à saúde, bem como o processo de transição demográfico-epidemiológica que vem ocorrendo no país nas últimas décadas, tem influenciado de maneira significativa o perfil nosológico da população brasileira. Observa-se, de um lado, que as possibilidades de maior intervenção sobre o meio ambiente contribuíram para reduzir as doenças infectocontagiosas, apesar de serem ainda, a quinta causa de mortalidade do país. Por outro lado, as repercussões do desenvolvimento científico e tecnológico nas condições de vida da população, têm levado ao aumento da expectativa de vida, expondo a população a um maior risco de desenvolver doenças crônico-degenerativas, ocupando estas, as primeiras posições nas estatísticas de mortalidade do Brasil.

Pode-se assim identificar um padrão de morbi-mortalidade em que coexistem as doenças típicas do subdesenvolvimento com as de países desenvolvidos, retratando a influência dos contrastes ecônomico-sociais vigentes em nossa sociedade.

IDE \& CHAVES lembram que as doenças crônicas não resultam exclusivamente no processo de industrialização, pois algumas delas já eram identificadas desde a Antiguidade. Ressaltam, porém, como fato novo, o seu fantástico crescimento nos últimos 100 anos no mundo inteiro e no Brasil, nas últimas quatro décadas, com a aceleração do desenvolvimento industrial e urbano.

As doenças crônico-degenerativas relacionam-se, portanto, às condições de vida, trabalho e consumo da população, "gerando atenções psicossociais e, conseqüentemente, o desgaste e a deterioração orgânico-funcional, com especial sobrecarga dos sistemas nervoso, endócrino e cardiovascular". Neste contexto, um número cada vez maior de indivíduos com este padrão de afecções tende a compor a clientela dos serviços de saúde.

Historicamente, uma das definições de doença crônica mais amplamente aceita é aquela proposta em 1957 pela Comissão de Doenças Crônicas de Cambridge, na qual se incluíam todos os desvios do normal que tinham uma ou mais das seguintes características: permanência, presença de incapacidade residual, mudança patológica não reversível no sistema corporal, necessidade de treinamento especial do paciente para a reabilitação e previsão de um longo período de supervisão, observação e cuidados.

A doença crônica pode começar como uma condição aguda, aparentemente insignificante e que se prolonga através de episódios de exacerbação e remissão. Embora seja passível de controle, o acúmulo de eventos e as restrições impostas pelo tratamento podem levar a uma drástica alteração no estilo de vida das pessoas.

A este respeito, TRENTINI et al observaram que, a partir do diagnóstico de doença crônica, os indivíduos a cometidos passam a ter novas incumbências como fazer regime de tratamento, conhecer a doença e lidar com incômodos físicos; 
perdas nas relações sociais, financeiras, nas atividades como locomoção, trabalho e lazer, ameaças à aparência individual, à vida e à preservação da esperança.

A complexidade e a extensão da problemática inerente à vivência da cronicidade da doença têm levado muitos autores a desenvolverem estudos com o objetivo de analisar o impacto desta condição sobre a qualidade de vida das pessoas a cometidas por doença crônica, sobe diferentes aspectos.

A qualidade de vida é um conceito intensamente marcado pela subjetividade, envolvendo todos os componentes essenciais da condição humana, quer seja físico, psicológico, social, cultural ou espiritual.

Essa concepção holística do homem é o fundamento básico que permeia toda a assistência de enfermagem, lembrando que o doente, seja em unidade de internação ou ambulatorial, é uma pessoa e, como tal, não é um ser isolado, não abandona todo o seu contexto de vida depois de ser acometido pela doença. $O$ cuidado a ser prestado dependerá, entre outros fatores, da percepção que ele e seu grupo familiar têm da doença e do significado que a experiência tem para eles.

O convívio com pacientes acometidos por doenças crônicas durante o estágio em Clínica Médica, trouxe à tona questionamentos para os quais necessitávamos de respostas a fim de melhor fundamentar a assistência a eles prestada.

Decidimos, assim, realizar este trabalho, com os seguintes objetivos:

- Identificar o que significa qualidade de vida para as pessoas com doença crônica;

- Verificar a interferência da doença crônica da doença crônica sobre a qualidade de vida destas pessoas.

\section{II - METODOLOGIA}

O presente estudo foi desenvolvido nos ambulatórios de Clínica Médica de dois hospitais governamentais de ensino da cidade de São Paulo.

\section{1 - População de estudo:}

Foi composta por 71 pacientes de ambos os sexos com idade entre 13 e 79 anos, em seguimento ambulatorial nos hospitais acima referidos.

\section{2 - Critérios de Seleção:}

Foram incluídos no presente estudo, as pessoas que atenderam os seguintes requisitos:

- apresentar doença crônica de qualquer natureza;

- contactar verbalmente; 
- manifestar interesse em participar do estudo;

- estar presente nos ambulatórios nos dias úteis da semana, das 14 às 18 horas. Estes períodos foram definidos em função da disponibilidade dos pesquisadores para a coleta de dados e da dinâmica de atendimento a pacientes crônicos nos serviços mencionados.

\section{3 - Coleta de Dados:}

A coleta de dados foi realizada nos meses de setembro e outubro de 1993. As entrevistas foram realizadas pelas alunas autoras do trabalho e tiveram a duração média de 15 minutos. As pessoas foram entrevistadas no momento em que aguardavam a consulta no ambulatório ou logo após o término da mesma.

\subsection{1 - Instrumento para a coleta de dados:}

Utilizamos como referencial teórico, as áreas pertinentes à qualidade de vida mencionadas nos trabalhos de BURCKHARDT et al; THOMPSON e TRENTINI et al. Foi utilizado para coleta de dados um formulário (Anexo 1) constando de 3 partes, com os seguintes dados:

Parte I: Identificação

Parte II: Diagnóstico e tratamento

Parte III: Significado de qualidade de vida; interferência da doença crônica na qualidade de vida de diferentes aspectos: trabalho/estudo/atividades do lar, relacionamento familiar e com amigos, recreação e lazer, capacidade física, independência/auto-cuidado e auto-sistema.

O formulário incluiu perguntas abertas e fechadas, destinadas a obtenção dos dados subjetivos relacionados ao tema, permitindo a livre verbalização por parte do respondente.

Foi realizado um pré-teste com o objetivo de verificar a melhor forma de entrevistar as pessoas e identificar possíveis falhas na formulação das questões e no registro dos dados. Realizamos 10 entrevistas para avaliação do instrumento e após os ajustes necessários, procedemos à coleta de dados.

\subsection{2 - Operacionalização da coleta de dado:}

A coleta de dados foi iniciada após a autorização dos responsáveis pelas unidades ambulatoriais selecionadas para o estudo.

Os entrevistados foram identificados através do prontuário, onde constavam o diagnóstico médico e os dados de identificação, os quais eram validados e complementados no momento da entrevista.

No primeiro contato, esclarecemos quanto aos objetivos do estudo, garantindo às pessoas o direito de não participação e o anonimato das informações fornecidas. 
Para se conseguir maior fidelidade possível, procuramos registrar, na íntegra as declarações das pessoas a respeito de suas experiências e percepções sobre o assunto. Foi necessário, às vezes, estimulá-las a falar, tomando-se o cuidado de não induzir as respostas.

\subsection{3 - Análise dos dados:}

As informações contidas nos formulários foram computadas manualmente e analisadas segundo freqüência absoluta e relativa (porcentagem). Os dados provenientes das questões abertas foram categorizadas e analisados segundo a sua natureza.

\section{III - RESULTADOS E COMENTÁRIOS}

\section{1 - Dados de identificação:}

Participaram do estudo 71 pessoas, das quais 19 eram do sexo masculino $(26,8 \%)$ e $52(73,2 \%)$, do sexo feminino.

A idade das pessoas variou entre 13 e 79 anos, sendo que o grupo etário de maior freqüência, tanto para o sexo masculino como para o sexo feminino foi o de 50 a 70 anos $(68,4 \%$ e $42,3 \%$ respectivamente). Isto se explica, talvez, pelo fato de que, com o aumento da idade, aumente também a freqüência de problemas crônicos.

Com relação ao estado civil, verificamos o predomínio de pessoas casadas $(59,1 \%)$, encontrando-se estas predominantemente na faixa etária de 30 a 70 anos. Incluíram-se nesta categoria, 2 pessoas que informaram ser amasiadas.

Em relação ao nível de instrução, o primeiro grau incompleto teve a maior freqüência, com 40 pessoas $(56,3 \%)$, enquanto que $12(16,9 \%)$ informaram ter 0 primeiro grau completo. Verificamos também que 7 (9,9\%) eram analfabetas.

Para a análise das ocupações utilizou-se o modelo proposto por FONSECA, que as classifica em níveis, com algumas adaptações para este trabalho:

nível I - cargos de ocupação manual não especializada, que inclui pessoas encarregadas de executar tarefas que não exigem experiência profissional prévia.

nível II - cargos de ocupação manual especializada, que inclui pessoas encarregadas de executar tarefas artesanais e manuais, requerem compreensão dos processos que intervém no trabalho, além de habilidade manual.

nível III - cargos de supervisão, gerência e outras ocupações não manuais.

nível IV - profissional liberal e cargos de alta administração.

nível V - sem ocupação definida (estudante, desempregado, aposentado).

Observamos, assim, a predominância das ocupações de nível I (46,5\%), 
seguidas de nível V $(29,6 \%)$, nível III (9,9\%), e de níveis II e IV $(7,0 \%)$.

Quanto à renda familiar, observou-se que a maioria das pessoas $(56,5 \%)$ contava com uma renda entre 1 e 2 salários mínimos (SM), sendo que $8,1 \%$ sobrevivia com menos de $1 \mathrm{SM}$.

Estes dados, associados àqueles relativos ao nível de instrução e ocupação, ratificam a caracterização de uma clientela de baixo nível instrucional e sócioeconômico.

No que se refere ao número de dependentes, $33,8 \%$ dos entrevistados não tinham dependentes, provavelmente pelo fato de que a maioria da população estava inserida na faixa etária de 50 a 70 anos, idade em que, geralmente, os filhos não mais dependem financeiramente dos pais. Verificamos também que $23,9 \%$ das pessoas possuíam 1 dependente e 19,7\%, 3 dependentes.

Quanto aos dados relativos à naturalidade, $50,7 \%$ da população era natural da região sudeste, mas pudemos notar um grande contingente de nordestinos (39,4\%).

Com relação ao tempo de residência em São Paulo, 67,5\% dos entrevistados moravam em São Paulo há mais de 20 anos.

\section{2 - Dados de diagnóstico e tratamento:}

Os tipos de doença crônica mais freqüentemente encontrados na população pesquisada estão apresentados na Figura 1.

Figura 1 - Distribuição da população segundo tipo de doença crônica.

São Paulo, 199

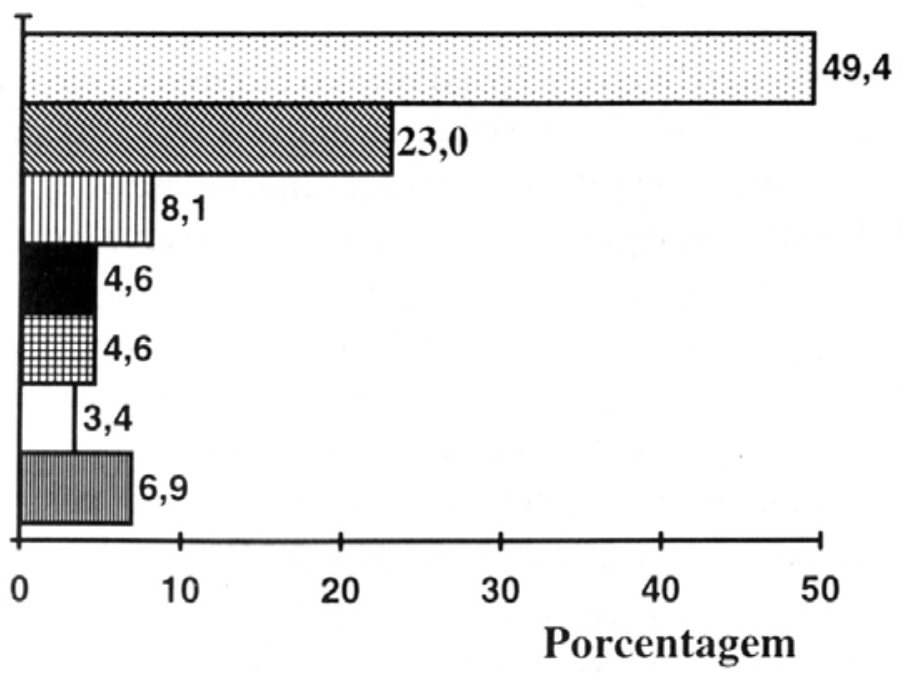

口Hipertensão Arterial
$\mathbb{N}$ Diabetes Mellitus
四Asma Brônquica
Artrite Reumatóide
田Bronquite
$\square$ Cirrose Hepática
$\square$ Outros

Os dados da figura 1 são compatíveis com a característica de atendimento dos ambulatórios onde os dados foram coletados. Em ambos os serviços funcionam Ligas de Hipertensão e Diabetes, razão pela qual estes foram os tipos de afecções mais encontrados $(49,4 \%$ e $23,0 \%$, respectivamente). Pessoas com doenças respiratórias crônicas como asma e bronquite também compuseram a população de 
estudo, num percentual de $12,7 \%$, assim como os portadores de artrite reumatóide $(4,6 \%)$ e cirrose hepática $(3,4 \%)$. Algumas pessoas apresentaram mais de um diagnóstico, sendo mais freqüente a associação de hipertensão arterial e diabetes, fato que originou a identificação de 87 diagnósticos entre as 71 pessoas do estudo.

Com relação aos sinais e sintomas mais freqüentes na população, cefaléia, fadiga/cansaço e dor em diferentes partes do corpo foram os mais citados, representados individualmente por $11,2 \%$, seguidos de vertigem/lipotímia $(8,4 \%)$, dispnéia $(8,0 \%)$ e fraqueza/desânimo $(6,1 \%)$.

Com relação à distribuição da população segundo tempo de diagnóstico, grande parte dos entrevistados $(40,2 \%)$ conhecia seu diagnóstico há 10 anos ou mais, vindo em seguida o grupo que o conhecia entre 4 a 6 anos (18,2\%).

Das 71 pessoas, $44(62,0 \%)$ informaram nunca terem sido internados em decorrência de doenças crônicas. Das 27 pessoas $(38,0 \%)$ com intenção prévia, a maioria $(66,7 \%)$ informou ter sido internada de 1 a 2 vezes. Observamos também que 5 destas pessoas $(18,5 \%)$ tiveram 7 ou mais internações.

Os motivos de internação relacionaram-se à agudização dos problemas crônicos, acompanhados ou não de diferentes sinais e sintomas inespecíficos como: mal estar, nervosismo, fraqueza, cansaço, cefaléia, entre outros. Assim, as causas mais freqüentes de internação foram crise hipertensiva, descompensação diabética, crise asmática. As pessoas necessitaram, ainda, de internação para realizar exames e intervenções cirúrgicas (amputação do hálux, revascularização de membros inferiores).

\section{3 - Dados sobre qualidade de vida:}

Os significados atribuídos à qualidade de vida foram categorizados em diferentes aspectos, conforme demonstrado na Tabela 1.

Tabela 1 - Aspectos de qualidade de vida mencionados pelas pessoas entrevistadas. São Paulo, 1993

\begin{tabular}{|c|c|c|}
\hline aspectos & $n^{\circ}$ & $\%$ \\
\hline bem-estar material & 77 & 40,9 \\
\hline bem-estar físico & 45 & 23,9 \\
\hline bem-estar emocional & 21 & 11,2 \\
\hline lazer / recreação & 12 & 6,4 \\
\hline relacionamento familiar / social & 11 & 5,8 \\
\hline desenvolvimento pessoal (profissional) & 9 & 4,8 \\
\hline higiene pessoal e ambiental & 5 & 2,7 \\
\hline bem-estar espiritual & 3 & 1,6 \\
\hline outros (segurança, sorte, independência) & 5 & 2,7 \\
\hline Total & 188 & 100,0 \\
\hline
\end{tabular}


As 71 pessoas entrevistadas indicaram 188 aspectos relacionados à qualidade de vida, o que dá uma média de 2,6 indicações por pessoa.

As freqüências da Tabela 1 demonstraram que a população pesquisada relaciona qualidade de vida principalmente ao bem-estar material (40,9\%), explicitando itens como ter moradia, alimentação, vestuário, segurança financeira e emprego. Este dado está certamente relacionado ao nível sócio-econômico dos entrevistados, dos quais $82,3 \%$ possuíam rendimentos familiares de até 3 salários mínimos.

Outro aspecto enfocado foi a necessidade de bem-estar físico $(23,9 \%)$ : ter saúde, freqüentemente associado com ausência da doença crônica ou de seu sintomas. Em um estudo psicométrico sobre qualidade de vida de adultos, americanos com doença crônica, observou-se que os entrevistados consideraram o "estar saudável" como sendo uma área importante para sua qualidade de vida.

O terceiro aspecto mais lembrado foi o bem-estar emocional $(11,2 \%)$. Nesta área incluíram-se a sensação de tranqüilidade, paz, amor, alegria, compreensão, despreocupação, como características importantes para se ter qualidade de vida.

Aspectos como lazer/recreação, relacionamento familiar e social e desenvolvimento pessoal, também foram mencionados, porém, em menor freqüência.

A razão de termos apenas 3 menções sobre bem-estar espiritual relacionase, talvez, ao fato de que nossa população tinha suas doenças controladas. Em geral, quando a pessoas se depara com uma descompensação da doença, com a presença de dor ou com a morte, apega-se facilmente a práticas religiosas como rezar, ir à missa, participar de grupos religiosos, conversar com Deus, entre outros, como estratégias para enfrentar o sofrimento e a dor ${ }^{7}$.

Estudos realizados por Campbell e Flangan, apud BURCKHARDT et $\mathrm{al}^{2}$, demonstraram que, para os americanos em geral, qualidade de vida pode ser dimensionado em 5 domínios: bem-estar físico e material; relações com outras pessoas; participação em atividades sociais, comunitárias e cívicas; desenvolvimento e realização pessoal; recreação. BURCKHARDT et al ${ }^{2}$, utilizando o mesmo instrumento dos autores citados (Quality of Life Scale - QOLS) em americanos doentes crônicos, perceberam que os domínios não se alteeraram, incluindo-se, porém dois novos: a necessidade de independência e a habilidade de cuidar-se.

A referência ao aspecto independência foi pequena em nosso trabalho (apenas uma menção), ao contrário do que ocorreu no estudo norte-americano em que apareceu com grande freqüência ${ }^{2}$. Este fato pode estar relacionado às diferenças culturais, já que na cultura norte-americana a independência parece ser mais valorizada do que na brasileira.

A figura 2, apresentada a seguir, demonstra a interferência da doença crônica em diferentes aspectos relacionados à qualidade de vida. 


\section{Figura 2 - Interferência da doença crônica em aspectos relacionados}

\section{à qualidade de vida. São Paulo, 1993}

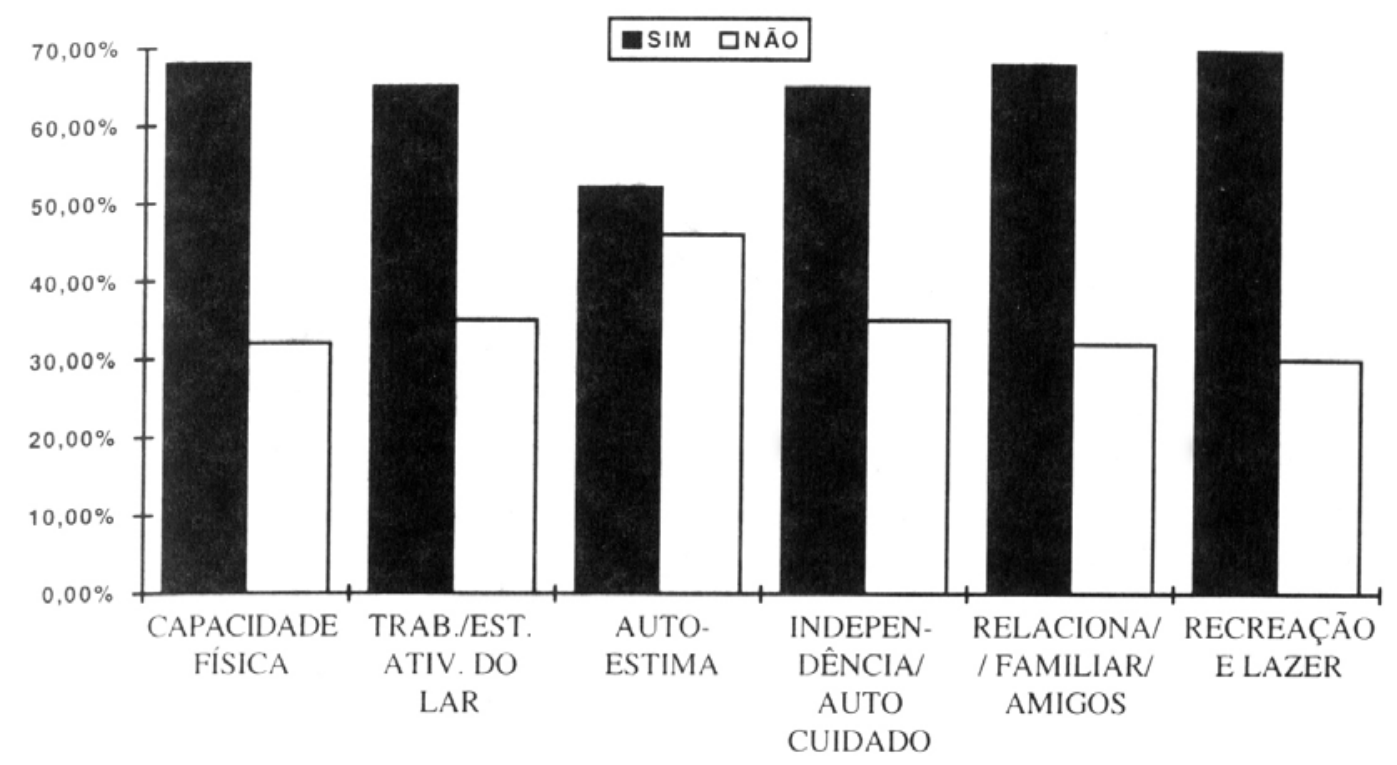

Verifica-se, nesta figura, que para os participantes do estudo, a doença crônica interferiu de maneira mais significativa na capacidade física, no trabalho/estudo/atividades do lar e na auto-estima.

Podemos, pensar, portanto, que de algum modo a qualidade de vida destas pessoas tenha sido afetada pela doença crônica, pois os aspectos acima mencionados estão intimamente ligados ao bem-estar material, físico e emocional, aspectos considerados pelos entrevistados como importantes para uma vida com qualidade (Tabela 1).

Os dados da Figura 2 indicam que 48 entrevistados $(67,6 \%)$ tiveram interferência da doença crônica na sua capacidade física, onde as limitações mais citadas foram andar, realizar esforço físico, carregar peso, subir escadas e correr. Estes resultados também podem ser encontrados em estudos como os de TRENTINI et al. ${ }^{10}$, em que a maioria dos informantes não tinha capacidade física para praticar esportes, para correr mais ou menos 300 metros quando com pressa, e para subir escadas de um piso a outro. Isto nos faz perceber que os indivíduos portadores de doenças crônicas deveram adaptar-se a várias mudanças. Muitas vezes o ambiente físico deve ser adaptado em decorrência da incapacidade física da pessoa; haverá também a necessidade de redimensionar o orçamento familiar visto que pode haver interferência da doenças nas atividades de trabalho da pessoa.

De fato, constatou-se que 46 pessoas $(64,8 \%)$ mencionaram ter ocorrido interferência da doença crônica no trabalho, estudos e atividades do lar. Referiram limitações nestas atividades devido à presença de sinais e sintomas, como por exemplo: cefaléia, tontura, dor, edema, fraqueza, dispnéia, tosse, mal-estar e alergia, levando à mudanças na carga horária e tipo de trabalho, ao afastamento temporário, à dificuldade para realizar atividades laborativas e a faltas escolares. 
Outro aspecto bastante mencionado foi a interferência da doença crônica sobre a auto-estima. As 38 pessoas $(53,5 \%)$ que mencionaram que esta interferência relacionaram-na à alteração do estado emociona, caracterizado por tristeza, desânimo, falta de vontade, desmotivação, nervosismo, aborrecimento, perda de prazer, insegurança, sensação de inutilidade e insatisfação com a auto-imagem.

Acreditamos que se houvesse um tempo maior, suficiente para estabelecer um relacionamento de confiança com a pessoa, a verbalização de sentimentos poderia ter sido um dado mais explorado e discutido.

Toda esta problemática vivenciada pela pessoa com doença crônica pode levar ao isolamento social, a um aumento da depressão e diminuição das expectativas de melhora, contribuindo para um sentimento de desesperança e solidão, os quais podem associar aos itens discriminados nos aspectos de recreação e lazer. Embora algumas pessoas não mencionassem este tipo de interferência, pois mesmo antes da doenças não tinham a recreação como hábito, 21 dos entrevistados $(29,6 \%)$ referiram tê-la abandonado, alegando problemas físicos (cansaço, cefaléia, dor nos membros, dificuldades para andar, dispnéia), emocionais (desânimo, medo, angústia, tristeza, preocupação, dependência de outros para sair, não ter prazer em se divertir, irritação com o lazer de outrem) e ambientais (intolerância a lugares quentes e fechados).

Os dados das 25 pessoas $(35,2 \%)$ que referiram a interferência da doença crônica na independência/autocuidado podem ser relacionados ao fato de que indivíduos com doenças crônicas possuem vários medos, entre eles, o medo da incapacidade, que gera a ansiedade pela possibilidade de tornar-se dependente de outrem para a satisfação das necessidades da vida diária. Foram também mencionadas as necessidades de auxílio no cuidado corporal, necessidade de companhia para sair e restrição e modificação de hábitos alimentares.

A maioria das pessoas relatou que não houve interferência no relacionamento familiar e com amigos de devido à doença crônica, porém, entre os que mencionaram ter ocorrido interferências $(32,4 \%)$, estas foram caracterizadas como desentendimentos com familiares por alterações de humor, impaciência e desequilíbrio emocional.

Embora em número bastante reduzido, houve indicações de que a doença crônica trouxe aspectos positivos como, por exemplo, o fato de passar a ter uma preocupação maior com a saúde e com o corpo ( 9 menções) e de receber maior atenção por parte dos familiares (3 menções).

\section{IV - CONCLUSÕES}

Para as pessoas com doença crônica incluída no presente estudo, o significado de qualidade de vida abrangeu, prioritariamente, os seguintes aspectos: 
bem-estar material (40,9\%), bem-estar físico (23,9\%), bem-estar emocional $(11,2 \%)$. Foram também mencionados, em menor freqüência, as áreas de recreação e lazer $(6,4 \%)$, o relacionamento familiar e social $(5,8 \%)$, o desenvolvimento pessoal $(4,8 \%)$, higiene pessoal e ambiental $(2,7 \%)$ e o bemestar espiritual (1,6\%). Outros aspectos como segurança, sorte e independência foram menos mencionadas $(2,7 \%)$.

A maioria das pessoas entrevistadas $(67,6 \%)$, referiu que a doença crônica alterou significativamente a sua capacidade física, sobretudo com relação à limitação para andar, no trabalho/estudo/atividades de lar, 64,8\% das pessoas referiram que a interferência foi no sentido de uma limitação destas atividades e da necessidade de parar de trabalhar devido a presença de diferentes sinais e sintomas; $53,5 \%$ das pessoas informaram interferências na auto-estima, relacionando-a com alteração do estado emocional. As áreas menos comprometidas pela doença crônica foram independência e autocuidado $(35,2 \%)$, relacionando-se à necessidade de auxílio no cuidado corporal, à necessidade de companhia para sair e à restrição e modificação de hábitos alimentares; relacionamento familiar e social (32,4\%), devido a desentendimentos com familiares e amigos por alterações do humor, impaciência e desequilíbrio emocional; recreação e lazer $(29,6 \%)$, no sentido de tê-los abandonado, alegando problemas físicos, emocionais e ambientais.

\section{V - CONSIDERAÇÕES FINAIS}

A realização do presente estudo nos possibilitou uma aproximação maior com a intensa e complexa problemática que envolve a vivência da doença crônica. Comprometendo muito além do que apenas a esfera biológica, ela acaba interferindo de diferentes formas no próprio estilo de vida das pessoas acometidas, do seu grupo familiar e social.

Foi importante constatar que a percepção da qualidade de vida, para os entrevistados, parece ser fortemente determinada por fatores de ordem sócioeconômica, uma vez que a doença crônica interrompe ou dificulta a inserção no processo produtivo, diminuindo as possibilidades de acesso aos bens de consumo.

Ao mesmo tempo, as pessoas com doença crônica passam também a experimentar diferentes sentimentos e comportamentos decorrentes de alterações na capacidade física, na auto-estima e na imagem corporal, nas relações com outras pessoas e na realização de uma série de atividades da vida diária.

Como profissionais envolvidos com a assistência à saúde, é de fundamental importância a consideração de que a doença seja ela aguda ou crônica, é uma experiência bastante complexa e individualizada. 
É preciso enfatizar também que, no processo de adaptação às limitações decorrentes da doença, nós enfermeiros, temos um importante papel a desempenhar. Neste processo muito há por se fazer no sentido de que as ações da equipe de saúde levem em conta a multidimensionalidade do conceito de qualidade de vida.

\section{QUALITY OF LIFE OF PEOPLE WITH CHRONIC ILLNESS}

The present study has the following objectives: to identify the significance of the quality of life people with chronic illness and verify its interference in their quality of life. We have studied 71 people of both sexes, attended in the clinical settings of 2 public hospitals. The most common diagnoses were high blood pressure and diabetes mellitus. The significance of quality of life to interviewed people was related mainly to material comfort (40,9\%); physical comfort $(23,9 \%)$ and emotional comfort $(11,2 \%)$. Chronic illness interfered in their quality of life because it has changed, especially, their physical capacity (67,6\%), their work/study/home activities $(64,8 \%)$ and their serfesteem (53,5\%). Based on the results, we emphasize the importance of nursing in the adaptation process of people and their relatives considering the limitations of chronic illness.

UNITERMS: quality of life, chronic illness.

\section{CALIDAD DE VIDA DE LA PERSONA CON ENFERMEDAD CRÓNICA}

El presente estudio tuvo como objetivos: identificar el significado de calidad de vida para la persona con enfermedad crónica y verificar la interferencia de la enfermedad sobre la calidad de vida de esas personas. Fueron entrevistados 71 personas de los dos sexos, atendidos en los ambulatorios de Clínica Médica de dos hospitales gubernamentales. Los diagnósticos más frecuentes fueron la hipertensión arterial y el diabetes mellitus. Se utilizó, para la recolección de los datos, un instrumento con preguntas abiertas y cerradas. El significado de calidad de vida para las personas entrevistadas se relacionó principalmente con bienestar material (40,9\%), bienestar físico (23,9\%) y bienestar emocional (11,2\%). La enfermedad crónica interfirió en la calidad de vida de las personas al modificar, sobretodo, su capacidad física (67,6\%), el trabajo/estudio/actividad en el hogar (64,8\%) y la autoestima (53,5\%). A partir de los resultados obtenidos, se enfatiza la importancia de 
considerar la multidimensionalidad del concepto de calidad de vida en la atención a la salud y en particular, en la atención de enfermería.

TÉRMINOS CLAVES: calidad de vida, enfermedad crónica.

\section{REFERÊNCIAS BIBLIOGRÁFICAS}

01. BRASIL. Ministério da Saúde. Fundação Nacional de Saúde. Centro Nacional de Epidemiologia. Coordenação de informação epidemiológica. Estatísticas de Mortalidade - Brasil 1987. Brasília, 1992.

02. BURCKHARDT, C.S. et al. Quality of life of adults with chronic illness: a psychometric study. Res. Nurs. Health, New York, v. 12, n. 6, p. 347-54, 1989.

03. FONSECA, G.T. Modelo para uma classificação de ocupações. Rev. Bras. Est. Pedag., v. 48, n. 107, p. 274-311, 1967.

04. GREGG, C.H.; ROBERTUS, J.L.; STONE, J.B. The psychological aspects of chronic illness. Springfield: Charles C. Thomas, 1989. cap. 1, p. 3-13.

05. IDE, C.A.C; CHAVES, E.C. A intervenção do enfermeiro na assistência ao diabético. Rev. Esc. Enfermagem USP. São Paulo, v. 26, n. 2, p. 187-204, 1992.

06. LUBKIN, I.M. Chronic illness: impact and interventions. 2 ed. Boston: Jones and Bartlett, 1990.

07. PIMENTA, C.A.M.; CRUZ, D.A.L.M. Câncer e dor: alterações nos padrões de resposta humana. Acta Paul. Enfermagem, São Paulo, v. 7, n. 1, p. 27-34, 1994.

08. THOMPSON, J.M. Quality of life after conventional ileostomy in New South Wales. WCET J. v. 10, n. 3, p. 8-14, 1990.

09. TRENTINI, M.: SILVA, D.G. da; LEIMANN, A.H. Mudanças no estilo de vida enfrentadas por pacientes em condições crônicas de saúde. Rev. Gauch. Enfermagem, Porto Alegre, v. 11, n. 1, p. 18-28, 1990.

10. TRENTINI, M. et al. Qualidade de vida dos indivíduos com doenças cardiovasculares crônicas e diabetes mellitus. Rev. Gauch. Enfermagem. Porto Alegre, v. 11 , n. 2, p. 20-7, 1990 


\section{ANEXO 1}

Formulário

I - Dados de Identificação:

Nome:

Idade:

Sexo:

Estado Civil:

Escolaridade: Ocupação/Profissão: Situação Empregatícia:

Renda Familiar: Número de Dependentes:

Naturalidade: Tempo de Residência em São Paulo:

II - Dados de Diagnósticos e tratamento:

Tipo de doença crônica: Sinais e sintomas apresentados:

Tempo de diagnóstico: Número de internações:

Motivo de internação:

III - Dados sobre Qualidade de Vida:

O que o(a) Sr(a) entende por Qualidade de Vida?

Houve interferência da doença crônica em relação aos seguintes aspectos?

- Trabalho/Estudos/Atividades do lar

Não Sim Como?

- Relacionamento Familiar/Amigos

Não Sim Como?

- Recreação / Laser

Não Sim Como?

- Capacidade Física

Não Sim Como?

- Independência / Auto-cuidado

Não Sim Como?

- Auto-estima

Não Sim Como? 